\title{
Deterministic Multi-Player Dynkin Games
}

\author{
Eilon Solan* and Nicolas Vieille ${ }^{\dagger \ddagger}$
}

September 3, 2002

\begin{abstract}
A multi-player Dynkin game is a sequential game in which at every stage one of the players is chosen, and that player can decide whether to continue the game or to stop it, in which case all players receive some terminal payoff.

We study a variant of this model, where the order by which players are chosen is deterministic, and the probability that the game terminates once the chosen player decides to stop may be strictly less than 1.

We prove that a subgame-perfect $\epsilon$-equilibrium in Markovian strategies exists. If the game is not degenerate, this $\epsilon$-equilibrium is actually in pure strategies, but in degenerate cases there need not exist a subgame-perfect $\epsilon$-equilibrium in pure strategies
\end{abstract}

*MEDS Department, Kellogg School of Management, Northwestern University, 2001 Sheridan Road, Evanston, IL 60208 and the School of Mathematical Sciences, Tel Aviv University. e-mail: e-solan@kellogg.northwestern.edu

${ }^{\dagger}$ Département Finance et Economie, HEC, 1, rue de la Libération, 78351 Jouy-enJosas, France. e-mail: vieille@hec.fr

${ }^{\ddagger}$ We acknowledge the financial support of the Arc-en-Ciel/Keshet program for 2001/2002. The research of the first author was supported by the Israel Science Foundation (grant No. 03620191). 


\section{Introduction}

Dynkin (1969) introduced the following zero-sum game of optimal stopping. The game involves two players, and two stochastic processes: $\left(i_{n}\right)_{n \in \mathbf{N}}$ is a $\{1,2\}$-valued process, which indicates which player is active at stage $n$, and $\left(r_{n}\right)_{n \in \mathbf{N}}$ is a $\mathbf{R}^{2}$-valued process, which indicates the terminal payoff.

At every stage $n$, the two players are informed of past and current values of the two processes. Player $i_{n}$, the active player at stage $n$, decides whether he continues or stops. The game stops at the first stage $\theta$ in which the active player chooses to stop. The payoff (paid by player 2 to player 1 ) is $r_{\theta}$ if $\theta<+\infty$ and zero otherwise. A pure strategy of player $i$ is a stopping time that is consistent with the rules of the game.

Dynkin proved that this game has a value if $\sup _{n \in \mathbf{N}}\left|r_{n}\right| \in L^{1}$, and constructed pure $\epsilon$-optimal strategies for the two players. Dynkin's $\epsilon$-optimal strategies are subgame-perfect in the sense that after every finite history, the continuation strategy is $\epsilon$-optimal in the subgame defined by that history.

An extensive literature developed from this seminal work. In a discrete time framework, much attention was paid to the case where the players are allowed to stop simultaneously. In the zero-sum case, several authors, including Kiefer (1971) and Neveu (1975), provided sufficient conditions for the existence of the value, when players are restricted to stopping times. Rosenberg et al. (2001) proved (under a minimal boundedness condition) that the value always exists, provided the players are allowed to use randomized stopping times. In the two-player non-zero-sum case, Shmaya and Solan (2002) proved that an $\varepsilon$-equilibrium always exists in randomized stopping times (again, under some boundedness condition).

In the present paper we consider a different generalization of Dynkin's model to a multi-player setup. Specifically, we analyze the following class of $I$-player games. A deterministic sequence $\left(i_{n}, p_{n}, r_{n}\right) \in I \times[0,1] \times \mathbf{R}^{I}$ is given. At each stage $n$ player $i_{n}$ chooses whether to continue or to stop. If he continues, the game continues to the next stage, while if he stops a lottery is performed. With probability $p_{n}$ the game terminates, yielding the payoff $r_{n}$, while with probability $1-p_{n}$ the game continues.

This model is more restrictive than the natural generalization of Dynkin's model, in that the order in which players alternate is deterministic. On the other hand, it incorporates an additional degree of generality, since a player may not be able to stop the process for sure at a given stage.

Our model can be used to analyze situations of shrinking markets (see, 
e.g., Ghemawat and Nalebuff (1985), Fine and Li (1989)), in which $n$ firms have to decide when to exit a shrinking market. Since once a firm exits, we remain with a market with $n-1$ firms, which can be solved inductively, we are reduced to a Dynkin game.

A similar situation occurs in takeover games, where $n$ firms strategically decide to make a takeover attempt on opponent firms. Since a takeover attempt is not always successful, the probability of termination upon stopping (=making a takeover attempt) may be strictly smaller than 1 .

In both of these models, the assumption that the order of players is deterministic can be explained by having different days of the week for board meetings of the various firms.

Another model which is close to the one we study is multi-player duels, or $n$-uels (see, e.g., Kilgour (1975, 1977) or Kilgour and Brams (1997)). In this model, $n$ gunners alternately have the option to shoot one of their opponents or abstain. Since once a gunner hits one of his opponents we are left with a game with $n-1$ players, which can be solved inductively, the game is essentially reduced to a deterministic Dynkin game where players have several stop actions. As the accuracy of the gunners may not be perfect, the probability of termination upon stopping (=shooting) may be strictly less than 1 .

Our main result states that if the sequence $\left(r_{n}\right)$ of payoffs is bounded, a subgame-perfect $\varepsilon$-equilibrium in Markovian strategies exists. Moreover, unless the game is degenerate, this $\varepsilon$-equilibrium is in pure strategies. However, in degenerate cases, a subgame-perfect 0-equilibrium need not exist. Since the subgame-perfect $\epsilon$-equilibrium we identify is in Markovian strategies, it is robust to the information players receive along the game; all they need to know is the stage of the game. Translated to the $n$-uel model, this means that there is a subgame-perfect $\epsilon$-equilibrium which is also a subgame-perfect $\epsilon$-equilibrium in the silent $n$-uel, in which players do not observe missed shots.

A multi-player Dynkin game is a game of perfect information. Hence, for every $\varepsilon>0$, by Mertens' (1987) generalization of Martin's (1975) result, there is a Nash $\varepsilon$-equilibrium. However, this $\varepsilon$-equilibrium may involve noncredible threats of punishment.

The paper is arranged as follows. In Section 2 we present the model and the main result. Several examples appear in Section 3. The proof of the main result appears in Section 4. The paper concludes in Section 5 with a discussion and open problems. 


\section{The Model and the Main Result}

\subsection{Multi-player deterministic Dynkin games}

A deterministic multi-player Dynkin game $\Gamma=\left(I,\left(i_{n}, p_{n}, r_{n}\right)_{n \in \mathbf{N}}\right)$ is given by

- A finite set $I$ of players.

- For every $n \in \mathbf{N}$, a triplet $\left(i_{n}, p_{n}, r_{n}\right) \in I \times[0,1] \times \mathbf{R}^{I}$.

The triplet $\left(i_{n}, p_{n}, r_{n}\right)$ specifies who is allowed to stop at stage $n$, the probability of success in that case, and the corresponding payoff respectively.

The game is played in stages. At each stage $n \in \mathbf{N}$, provided the game has not terminated yet, player $i_{n}$ has to choose whether to continue or stop. If he decides to continue, the game continues to stage $n+1$. If he decides to stop, a lottery takes place (all lotteries in the game, including random choices by the players, are independent). With probability $p_{n}$ the game terminates, and the terminal payoff for the players is given by the vector $r_{n}$. With probability $1-p_{n}$ the game continues to stage $n+1$. If the game never terminates, the payoff is zero for each player.

We denote by $\theta$ the termination stage of the game, i.e., the first stage in which a player decides to stop, and the game terminates. Thus, the payoff to player $i \in I$ is $r_{\theta}^{i} \mathbf{1}_{\theta<\infty}$.

\subsection{Strategies and results}

A strategy of player $i \in I$ maps the set of information sets of player $i$ to the set of mixed moves of player $i$. We let $N_{i}=\left\{n \in \mathbf{N} \mid i_{n}=i\right\}$ be the set of stages in which player $i$ is active.

We are going to restrict the players to Markovian strategies; namely, strategies that depend only on the stage, and not on the history. We will prove below that the game admits a subgame-perfect $\varepsilon$-equilibrium in Markovian strategies. By a general observation (see, e.g., Fudenberg and Tirole (1991, p.501)), this subgame-perfect $\varepsilon$-equilibrium remains a subgame-perfect $\varepsilon$-equilibrium without the restriction to Markovian strategies.

In the present context, a (behavior Markovian) strategy of player $i$ is a function $\sigma^{i}: N_{i} \rightarrow[0,1]$, where $\sigma^{i}(n)$ is the probability assigned by player $i$ to stop at stage $n$, provided the game does not terminate before that stage. We denote the set of strategies of player $i$ by $\Sigma^{i}$. 
A strategy profile (or simply a profile) is a vector $\sigma=\left(\sigma^{i}\right)_{i \in I}$ of strategies, one for each player.

Every strategy profile $\sigma \in \times_{i \in I} \Sigma^{i}$ induces a probability distribution $\mathbf{P}_{\sigma}$ over the space of plays, or infinite histories. The corresponding expectation operator is $\mathbf{E}_{\sigma}$. Thus, the expected payoff to player $i$ given a strategy profile $\sigma$ is

$$
\gamma^{i}(\sigma):=\mathbf{E}_{\sigma}\left[r_{\theta}^{i} \mathbf{1}_{\theta<\infty}\right] .
$$

Before we state our result, we first recall standard equilibrium notions.

Definition 1 Let $\varepsilon \geq 0$. A strategy profile $\sigma$ is an $\varepsilon$-equilibrium if for every player $i \in I$ and every strategy $\tau^{i} \in \Sigma^{i}$,

$$
\gamma^{i}(\sigma) \geq \gamma^{i}\left(\sigma^{-i}, \tau^{i}\right)-\varepsilon .
$$

We mention that, for any $\varepsilon^{\prime}>\varepsilon$, an $\varepsilon$-equilibrium is a uniform $\varepsilon^{\prime}$-equilibrium; that is, it is a $\varepsilon^{\prime}$-equilibrium (a) in every discounted game, provided the discount factor is sufficiently small, and (b) in every $N$-stage game, provided $N$ is sufficiently large. Indeed, the proof provided in Solan and Vieille (2001, Proposition 2.13) does adapt to the present framework.

For $n \in \mathbf{N}$, we denote by

$$
\gamma_{n}(\sigma)=\mathbf{E}_{\sigma}\left[r_{\theta} \mathbf{1}_{\theta<\infty} \mid \theta \geq n\right]
$$

the conditional expected payoff, given that termination does not occur prior to stage $n$. Equivalently, $\gamma_{n}(\sigma)$ is the payoff induced by the strategy profile $\sigma$ in the subgame starting at stage $n$.

A strategy profile is a subgame-perfect $(\varepsilon$-)equilibrium of a game if it induces an $(\varepsilon-)$ equilibrium in any subgame. In the present context, this amounts to the following definition.

Definition 2 Let $\varepsilon \geq 0$. A strategy profile $\sigma$ is a subgame-perfect $\varepsilon$-equilibrium if for every $n \in \mathbf{N}$, every player $i \in I$, and every $\tau^{i} \in \Sigma^{i}$,

$$
\gamma_{n}^{i}(\sigma) \geq \gamma_{n}^{i}\left(\sigma^{-i}, \tau^{i}\right)-\varepsilon
$$

Our main result is the following.

Theorem 3 Let $\Gamma=\left(I,\left(i_{n}, p_{n}, r_{n}\right)_{n \in \mathbf{N}}\right)$ be a deterministic Dynkin game. If the sequence $\left(r_{n}\right)_{n \in \mathbf{N}}$ is bounded, then for every $\varepsilon>0$, the game $\Gamma$ admits a subgame-perfect $\varepsilon$-equilibrium in Markovian strategies. 
We conclude this section with two comments.

As will be clear from the proof, in most cases, there is a pure subgameperfect equilibrium. However, this is not always true (see Example 3 below). This is in sharp contrast with finite extensive games of perfect information and with two-player zero-sum Dynkin games.

Our proof is valid as long as $\gamma(\sigma)$ is uniformly bounded, for every profile $\sigma$ (which is the case when the sequence $\left(r_{n}\right)_{n \in \mathbf{N}}$ is bounded.) If this does not hold, the payoff function of the game is not well-defined.

\section{$3 \quad$ Examples}

In the present section we provide several examples, that illustrate the main features of the model.

Example 1: Take $I=\{1,2,3\}$ and

$$
\left(i_{n}, p_{n}, r_{n}\right)= \begin{cases}(1,1,(1,0,3)) & n=1 \text { modulo } 3 \\ (2,1,(3,1,0)) & n=2 \text { modulo } 3 \\ (3,1,(0,3,1)) & n=0 \text { modulo } 3\end{cases}
$$

In words, at the first stage, player 1 can stop the game, thereby yielding the payoff vector $(1,0,3)$. If player 1 chooses to continue, at the second stage player 2 can stop the game, yielding the terminal payoff $(3,1,0)$. If player 2 chooses to continue as well, at the third stage player 3 can stop the game, yielding the terminal payoff $(0,3,1)$. The process then repeats itself cyclically. This game is a variation upon a game studied by Flesch et al. (1997).

We will characterize all pure subgame-perfect 0-equilibrium profiles of that game, using backward induction.

Let $\sigma$ be such a 0 -equilibrium. Assume that at stage $3 n$, for some $n \geq 2$, player 3 stops with probability 1 ; that is, $\sigma^{3}(3 n)=1$. In particular, $\gamma_{3 n}(\sigma)=$ $(0,3,1)$.

Consider the subgame starting at stage $3 n-1$. In that subgame, player 2 receives $\gamma_{3 n}^{2}(\sigma)=3$ if he chooses to continue at stage $3 n-1$, while he receives only 1 if he chooses to stop. By the subgame-perfect equilibrium condition, player 2 continues at stage $3 n-1$, that is, $\sigma^{2}(3 n-1)=0$. Hence $\gamma_{3 n-1}(\sigma)=\gamma_{3 n}(\sigma)=(0,3,1)$. 
We repeat this argument with the subgame starting at stage $3 n-2$. By continuing at stage $3 n-2$ player 1 receives 0 , as the game will be terminated at stage $3 n$, while by stopping he receives 1 . By the subgame-perfect equilibrium condition, $\sigma^{1}(3 n-2)=1$ and $\gamma_{3 n-2}(\sigma)=(1,0,3)$.

Applying this backward induction argument repeatedly, we get that $\sigma^{3}(3 n-$ $3)=0, \sigma^{2}(3 n-4)=1, \sigma^{1}(3 n-5)=0$ and $\sigma^{3}(3 n-6)=1$. The cycle of length 6 then repeats itself.

On the other hand, if $\sigma^{3}(3 n)=0$ for some $n \geq 2$, then $\sigma^{3}(3 n-3)=1$ and the previous analysis holds.

Thus, there are two pure subgame-perfect 0-equilibria: (a) at odd stages the active player stops, and at even stages the active player continues, and (b) at even stages the active player stops, and at odd stages the active player continues.

Remark 4 This game admits other subgame-perfect equilibria. In particular, the profile in which each player stops with probability $1 / 2$ whenever active, is a subgame-perfect equilibrium. In a sense, it corresponds to the 0-equilibria constructed by Flesch et al. (1997).

In the next example, we allow for probabilities of success below one.

Example 2: Consider the following modification of Example 1, where $I=\{1,2,3\}$, and

$$
\left(i_{n}, p_{n}, r_{n}\right)= \begin{cases}(1,1,(1,0,3)) & n=1 \text { modulo } 3 \\ (2,1 / 2,(3,1,0)) & n=2 \text { modulo } 3 \\ (3,1 / 2,(0,3,1)) & n=0 \text { modulo } 3\end{cases}
$$

Thus, when player 1 stops the game terminates with probability 1 , while when either player 2 or player 3 stops the game terminates with probability $1 / 2$.

As we did in Example 1, we characterize the set of subgame-perfect 0equilibrium in pure strategies. Let $\sigma$ be such a strategy profile. Let $n>0$ and $i$ be the active player at stage $n$. By the subgame-perfect equilibrium condition, $\sigma^{i}(n)=1$ if $\gamma_{n+1}^{i}(\sigma)<1$ and $\sigma^{i}(n)=0$ if $\gamma_{n+1}^{i}(\sigma)>1$.

Let $n \geq 3$, and assume that $\sigma^{1}(3 n+1)=1$. Then $\gamma_{3 n+1}(\sigma)=(1,0,3)$, and therefore $\sigma^{3}(3 n)=0$. This implies that $\gamma_{3 n}(\sigma)=\gamma_{3 n+1}(\sigma)=(1,0,3)$, and therefore $\sigma^{2}(3 n-1)=1$. 
It follows that

$$
\gamma_{3 n-1}(\sigma)=\frac{1}{2}(3,1,0)+\frac{1}{2}(1,0,3)=\left(2, \frac{1}{2}, \frac{3}{2}\right),
$$

and therefore $\sigma^{1}(3 n-2)=\sigma^{3}(3 n-3)=0$ and $\sigma^{2}(3 n-4)=1$.

Then

$$
\gamma_{3 n-4}(\sigma)=\frac{1}{2}(3,1,0)+\frac{1}{2}\left(2, \frac{1}{2}, \frac{3}{2}\right)=\left(\frac{5}{2}, \frac{3}{4}, \frac{3}{4}\right),
$$

and therefore $\sigma^{1}(3 n-5)=0$ and $\sigma^{3}(3 n-6)=1$.

One therefore has

$$
\gamma_{3 n-6}(\sigma)=\frac{1}{2}(0,3,1)+\frac{1}{2}\left(\frac{5}{2}, \frac{3}{4}, \frac{3}{4}\right)=\left(\frac{5}{4}, \frac{15}{8}, \frac{7}{8}\right),
$$

and therefore $\sigma^{2}(3 n-7)=0, \sigma^{1}(3 n-8)=0$ and $\sigma^{3}(3 n-9)=1$.

Finally,

$$
\gamma_{3 n-9}(\sigma)=\frac{1}{2}(0,3,1)+\frac{1}{2}\left(\frac{5}{4}, \frac{15}{8}, \frac{7}{8}\right)=\left(\frac{5}{8}, \frac{39}{16}, \frac{15}{16}\right),
$$

and therefore $\sigma^{2}(3 n-10)=0$, and $\sigma^{1}(3 n-11)=1$.

Therefore, any pure subgame-perfect 0 -equilibrium must repeat the sequence (starting with player 1$)(1,0,1 ; 0,0,1 ; 0,1,0 ; 0,1,0)$ of mixed moves. Along this cycle, player 1 first stops, then player 3 stops twice in a row, then player 2 stops twice in a row. This difference with the subgame-perfect 0-equilibrium of Example 1 arises since the probability of termination is here below one. By decreasing further the probabilities $p_{n}$ (but keeping $p_{n}=1$ for $n=1 \bmod 3$ ), one can create examples in which all pure subgame-perfect equilibria have cycles of arbitrary length.

We next introduce a two-player game that has no subgame-perfect 0equilibrium and no pure subgame-perfect $\varepsilon$-equilibrium.

Example 3: Take $I=\{1,2\}$, and

$$
\left(i_{n}, p_{n}, r_{n}\right)= \begin{cases}(1,1,(-1,2)) & n \text { is odd } \\ (2,1,(-2,1)) & n \text { is even }\end{cases}
$$

Fix $\varepsilon \in(0,1)$, and let $\sigma$ be the strategy profile defined by $\sigma^{1}(2 n+1)=1$ and $\sigma^{2}(2 n+2)=\varepsilon$ for every $n \geq 0$. We claim that $\sigma$ is a subgame-perfect $\varepsilon$-equilibrium. One should verify that player 1 (resp. player 2 ) cannot profit 
by deviating in the subgames that start at odd (resp. even) stages. Consider first the subgame that starts at stage $2 n+1$, for some $n \geq 0$. By stopping at stage $2 n+1$ player 1 receives -1 , while, since player 2 eventually stops with probability 1 , player 1's payoff is at most -1 , whatever he plays. In the subgame starting at stage $2 n+2$, player 2's expected payoff under $\sigma$ is $\varepsilon+2(1-\varepsilon)=2-\varepsilon$, whereas the maximal payoff to player 2 in the game is 2 .

We next prove that the game has no subgame-perfect $\varepsilon$-equilibrium in pure strategies. Assume to the contrary that there exists such a profile $\sigma$.

We first claim that there is an infinite set of even stages in which player 2 chooses to stop. Otherwise, let $N$ be the maximal integer such that player 2 stops at stage $2 N$ (set $N=0$ if player 2 never stops). Consider now the subgame that starts at stage $2 N+2$. Since $\sigma$ is a subgame-perfect $\varepsilon$ equilibrium, this implies that under $\sigma$ player 1 never stops in this subgame: by never stopping he receives 0 , while by stopping he receives -1 . But this implies that under $\sigma$ player 2 stops in this subgame: by never stopping he receives 0 , while by stopping he receives 1 . This, however, contradicts the definition of $N$.

We next claim that there is at most one even stage in which player 2 chooses to stop. Together with the previous paragraph, this shows that there cannot be a subgame-perfect $\varepsilon$-equilibrium. By the preceding paragraph, there is an infinite sequence of even stages in which player 2 stops. Assume that player 2 stops at stage $2 N$, with $N>1$. Since $\sigma$ induces an $\varepsilon$-equilibrium in the subgame that starts at stage $2 N-1$, and since player 2 stops at stage $2 N$, under $\sigma$ player 1 stops at stage $2 N-1$. However, since player 1 stops at stage $2 N-1$, under $\sigma$ player 2 continues in all stages $2 k$ for $k<N$ : by continuing in all these stages he receives 2 , while his payoff upon stopping is 1 .

This example shows that pure subgame-perfect $\varepsilon$-equilibria need not exist. Such a case may arise when there is a player $i$ who by stopping gives everyone else high payoff, but he himself receives low payoff. It is then in the interest of his opponents to threaten him that if he does not stop, one of them will eventually stop and punish player $i$. The punisher, however, stops with low probability, so that player $i$ has a chance to correct his behavior and stop the game in a later stage.

We finally prove that there is no subgame-perfect 0-equilibrium. We 
argue by contradiction, and we let $\sigma$ be a subgame-perfect 0 -equilibrium. For $i=1,2$, we denote by $c^{i}$ the strategy that always continues, i.e., $c^{i}(n)=0$ for each $n \in N_{i}$. Note first that, for each $n \in \mathbf{N}$, one has

$$
\mathbf{P}_{\sigma}(\theta<+\infty \mid \theta \geq n)=1 .
$$

Indeed, the sequence $\left(\mathbf{P}_{\sigma}(\theta<+\infty \mid \theta \geq n)\right)_{n \in \mathbf{N}}$ would otherwise decrease to zero, hence the sequence $\left(\gamma_{n}(\sigma)\right)_{n \in \mathbf{N}}$ would converge to zero, and player 2 would have a profitable deviation in the subgame starting at stage $n$, for $n$ large enough. By (1) the game terminates with probability 1, hence at least one of the players eventually stops with probability 1 :

$$
\begin{aligned}
& \mathbf{P}_{\sigma^{1}, c^{2}}(\theta<+\infty \mid \theta \geq n)=1 \text { for each } n \in \mathbf{N}, \text { or } \\
& \mathbf{P}_{c^{1}, \sigma^{2}}(\theta<+\infty \mid \theta \geq n)=1 \text { for each } n \in \mathbf{N} .
\end{aligned}
$$

If (2) holds, then $c^{2}$ is the best reply to $\sigma^{1}$ in all subgames, hence $\sigma^{2}=c^{2}$. Since the unique best reply of player 1 to $c^{2}$ is $c^{1}$, one gets $\sigma=\left(c^{1}, c^{2}\right)$ - a contradiction to (1).

If (3) holds, there are infinitely many even integers $n$ such that $\sigma^{2}(n)>0$. By optimality of $\sigma^{1}$, and since (3) holds, one has $\sigma^{1}(n-1)=1$ for any such $n$. Therefore, (2) holds - a contradiction.

\section{The Proof of Theorem 3}

In the present section we prove Theorem 3.

\subsection{Preliminaries}

In this subsection, we analyze few degenerate cases, and slightly rephrase the problem. The core of the proof of Theorem 3 is in subsection 4.3.

Let $\Gamma=\left(I,\left(i_{n}, p_{n}, r_{n}\right)_{n \in \mathbf{N}}\right)$ be a game. Since the sequence $\left(r_{n}\right)_{n \in \mathbf{N}}$ is bounded, we can assume w.l.o.g. that payoffs are bounded by 1 .

Let $\tilde{\Gamma}=\left(I,\left(i_{n}, p_{n}, \tilde{r}_{n}\right)_{n \in \mathbf{N}}\right)$ be another game with the same sequence of active players and the same probabilities of success. Since the payoff functions of the two games differ by at $\operatorname{most} \sup _{n \in \mathbf{N}}\left\|r_{n}-\tilde{r}_{n}\right\|$, any subgameperfect $\varepsilon$-equilibrium of $\tilde{\Gamma}$ is a subgame perfect $\varepsilon^{\prime}$-equilibrium of $\Gamma$, where $\varepsilon^{\prime}=\varepsilon+\sup _{n \in \mathbf{N}}\left\|r_{n}-\tilde{r}_{n}\right\|$. 
Since we are looking for an $\varepsilon$-equilibrium, there is no loss of generality in assuming that the range of the sequence $\left(r_{n}\right)_{n \in \mathbf{N}}$ is finite, and that if $(i, r)$ and $(j, \tilde{r})$ are two distinct elements in that range then $r^{k} \neq \tilde{r}^{k}$ for every $k \in I$.

Notice now that Theorem 3 will follow if we prove that there is a subgameperfect $\varepsilon$-equilibrium in some subgame of $\Gamma$. Indeed, the conclusion for $\Gamma$ will then follow by applying backward induction to the first stages of the game. Moreover, since finite extensive games with perfect information have pure subgame-perfect equilibria, the resulting profile will be pure when the subgame-perfect $\varepsilon$-equilibrium of the subgame is pure.

Let $I R$ be the finite range of the sequence $\left(i_{n}, r_{n}\right)_{n \in \mathbf{N}}$. For each $(i, r) \in I R$ define

$$
\pi(i, r)=\sum\left\{p_{n} \mid n \in \mathbf{N},\left(i_{n}, r_{n}\right)=(i, r)\right\}
$$

and set $I R_{0}=\{(i, r) \in I R \mid \pi(i, r)=+\infty\}$. If $\pi(i, r)=+\infty$ then if player $i$ stops whenever $\left(i_{n}, r_{n}\right)=(i, r)$, and all players continue in all other stages, the game will eventually terminate, and the terminal payoff will be $r$.

We now prove that we may assume w.l.o.g. that

$$
\pi(i, r)=0 \text { for each }(i, r) \notin I R_{0} .
$$

To see this, choose first $N \in \mathbf{N}$ large enough such that

$$
\sum_{n \geq N:\left(i_{n}, r_{n}\right)=(i, r)} p_{n}<\varepsilon /|I R| \text { for each }(i, r) \notin I R_{0}
$$

and denote by $\Gamma_{N}$ the subgame that starts at stage $N$. Let $\tilde{\Gamma}=\left(I,\left(i_{n}, \tilde{p}_{n}, r_{n}\right)_{n \in \mathbf{N}}\right)$ be the game that coincides with $\Gamma_{N}$ except that $\tilde{p}_{n}=0$ whenever $\left(\tilde{i}_{n}, \tilde{r}_{n}\right) \notin$ $I R_{0}$.

By (5), the payoff functions of the two games $\Gamma_{N}$ and $\tilde{\Gamma}$ differ by at most $\varepsilon$. Therefore, any subgame-perfect $\varepsilon$-equilibrium of $\tilde{\Gamma}$ is a subgame-perfect $2 \varepsilon$-equilibrium of $\Gamma_{N}$, and, by backward induction, yields a subgame-perfect $2 \varepsilon$-equilibrium of $\Gamma$.

By construction, the game $\tilde{\Gamma}$ satisfies (4). Therefore our claims holds.

Thus, we are led to analyze games such that, for each $(i, r) \in I R$, either $\pi(i, r)=0$ or $\pi(i, r)=+\infty$ holds. If $\pi(i, r)=0$ for each $(i, r) \in I R$, the payoff function of the game is identically zero and the conclusion of Theorem 3 follows trivially. Assume now that $\pi(i, r)=+\infty$ for some $(i, r) \in I R$. Consider the game obtained by dropping all stages $n$ such that $\pi\left(i_{n}, r_{n}\right)=0$ 
(and by relabeling stages). Since there are infinitely many stages $n$ such that $\pi\left(i_{n}, r_{n}\right)=+\infty$, the resulting game is again a multi-player deterministic Dynkin game. Plainly, any subgame perfect $\varepsilon$-equilibrium of this new game is also a subgame perfect $\varepsilon$-equilibrium of the initial game (with the proper identification of stages, and with an arbitrary behavior in the stages that have been dropped).

To conclude, we can therefore assume w.l.o.g. that $\pi(i, r)=+\infty$ for each $(i, r) \in I R$.

\subsection{A simple case}

Under the assumption that $\pi(i, r)=+\infty$ for each $(i, r) \in I R$, the proof proceeds by induction over the number of elements in $I R$. The conclusion is easy if $|I R|=1$, and is left to the reader.

We now analyze a somewhat degenerate case that generalizes Example 2.

This is the only place in the proof where we use the induction hypothesis.

Lemma 5 Assume that there exists $(i, r) \in I R$ such that

$$
r^{j} \geq \tilde{r}^{j} \text { for every }(j, \tilde{r}) \in I R .
$$

Then, for each $\varepsilon>0$, there is a subgame-perfect $\epsilon$-equilibrium.

The lemma states that if there is a terminal payoff $r$ that is preferred by each player $i$ to all terminal payoffs $i$ controls, then a subgame-perfect $\varepsilon$ equilibrium exists.

Proof. We split the discussion into three cases.

Case 1: $r^{i} \geq 0$.

Let $\sigma$ be the pure strategy profile in which player $i$ stops whenever $\left(i_{n}, r_{n}\right)=$ $(i, r)$, and all players continue in all other stages, i.e.,

$$
\sigma^{i_{n}}(n)=1 \text { if and only if }\left(i_{n}, r_{n}\right)=(i, r) .
$$

Fix $n \in \mathbf{N}$. We prove that $\sigma$ induces a 0 -equilibrium in the subgame that starts at stage $n$. Since $\pi(i, r)=+\infty$, the game eventually terminates, and therefore the expected payoff is $r$. Player $i$ cannot gain by deviating, since his payoff is at most $r^{i}$ if he terminates the game, and $0 \leq r^{i}$ if he 
always continues. Every player $j \neq i$ cannot gain by deviating either, since his payoff under $\sigma$ is $r^{j}$, while if he deviates his payoff is in the convex hull of $r^{j}$ and $\left\{\tilde{r}^{j},(j, \tilde{r}) \in I R\right\}$, hence at most $r^{j}$.

Case 2: $\quad r^{i}<0$, and there is $(j, \tilde{r}) \in I R$ such that $i \neq j$ and $\tilde{r}^{i}<r^{i}$.

Let $\left(n_{k}\right)_{k \in \mathbf{N}}$ be an increasing sequence of integers such that $\left(i_{n}, r_{n}\right)=(i, r)$ for $n=n_{k}, k \in \mathbf{N}$. For $k \in \mathbf{N}$, set $m_{k}=\inf \left\{n \geq n_{k}:\left(i_{n}, r_{n}\right)=(j, \tilde{r})\right\}$. (The sequence $\left(m_{k}\right)_{k \in \mathbf{N}}$ is non-decreasing but need not be increasing.) Since $\pi(i, r)=\pi(j, \tilde{r})=+\infty$, these two sequences are infinite.

Let $\sigma$ be the strategy profile in which player $i$ stops at all stages $n_{k}$, player $j$ stops with probability $\varepsilon$ at all stages $m_{k}$, and all players continue otherwise, i.e.,

$$
\sigma^{i_{n}}(n)= \begin{cases}1 & \text { if } n=n_{k}, k \in \mathbf{N} \\ \varepsilon & \text { if } n=m_{k}, k \in \mathbf{N} \\ 0 & \text { otherwise }\end{cases}
$$

The profile $\sigma$ is a subgame-perfect $2 \varepsilon$-equilibrium. Indeed, consider the subgame that starts at stage $n$. By the choice of the sequence $\left(m_{k}\right)_{k \in \mathbf{N}}$, one has $\left\|\gamma_{n}(\sigma)-r\right\| \leq 2 \varepsilon: \gamma_{n}(\sigma)$ is equal to $r$ if $n>m_{k_{0}}$ where $k_{0}=\inf \{k$ : $\left.n_{k} \geq n\right\}$, and is equal to $\varepsilon \tilde{r}+(1-\varepsilon) r$ otherwise. Notice that, under any unilateral deviation, the game terminates with probability one. Therefore, for each player $l$ and each strategy $\tau^{l} \in \Sigma^{l}$, the payoff $\gamma_{n}^{l}\left(\tau^{l}, \sigma^{-l}\right)$ to player $l$ by deviating from $\sigma^{l}$ to $\tau^{l}$ is in the convex hull of the set $\left\{r^{l}, \tilde{r}^{l}\right\} \cup\left\{\hat{r}^{l},(l, \hat{r}) \in I R\right\}$, hence is at most $r^{l}$.

Case 3: $\quad r^{i}<0$, and $\tilde{r}^{i} \geq r^{i}$ for every $(j, \tilde{r}) \in I R$ with $i \neq j$.

Consider the modified game where one sets $p_{n}=0$ whenever $\left(i_{n}, r_{n}\right)=$ $(i, r)$, or, alternatively, one drops all stages in which $\left(i_{n}, r_{n}\right)=(i, r)$.

By the induction hypothesis, this game admits a subgame-perfect $\varepsilon$ equilibrium $\sigma^{\prime}$. Extend $\sigma^{\prime}$ to a profile $\sigma$ in the original game, by instructing player $i$ to continue at all stages $n$ such that $\left(i_{n}, r_{n}\right)=(i, r)$.

It is easy to see that $\sigma$ is a subgame-perfect $\varepsilon$-equilibrium. Indeed, for every player $j \neq i$, any deviation in the original game yields the same expected payoff the deviation yields in the modified game. Since $\sigma^{\prime}$ is a subgameperfect $\varepsilon$-equilibrium, no player $j \neq i$ can profit more than $\varepsilon$ by deviating in the original game.

Moreover, under $\sigma^{\prime}$ the expected payoff to player $i$ is at least $\min \left\{0, \min _{(j, \tilde{r}), j \neq i} \tilde{r}^{i}\right\} \geq$ $r^{i}$. Therefore, if player $i$ deviates at stages $n$ such that $\left(i_{n}, r_{n}\right)=(i, r)$ he 
cannot profit, while if he deviates in other stages, he cannot profit more than $\varepsilon$, since $\sigma^{\prime}$ is a subgame-perfect $\varepsilon$-equilibrium.

\subsection{The general case}

In view of Lemma 5, Theorem 3 will follow from Proposition 6 below.

Proposition 6 Let $\Gamma$ be a deterministic multi-player Dynkin game. Assume that for every $(i, r) \in I R$, (i) $\pi(i, r)=+\infty$, and (ii) there is $(j, \tilde{r}) \in I R$ such that $\tilde{r}^{j}>r^{j}$. Then, for every $\varepsilon>0$, the game $\Gamma$ has a subgame-perfect $\varepsilon$-equilibrium in pure Markovian strategies.

We do not know whether a subgame-perfect 0-equilibrium exists or not. The rest of this section is devoted to the proof of the proposition.

As remarked at the beginning of Section 4.1, we can assume w.l.o.g. that for every $(i, r),(j, \tilde{r}) \in I R$, either $(i, r)=(j, \tilde{r})$, or $r^{k} \neq \tilde{r}^{k}$ for every $k$.

For every $i \in I$ set

$$
m^{i}=\max \left\{r^{i} \mid(i, r) \in I R\right\} .
$$

This is the maximal terminal payoff player $i$ can receive when he alone stops. Let $\rho_{i} \in \mathbf{R}^{I}$ be the unique vector $r$ such that $(i, r) \in I R$ and $r^{i}=m^{i}$ (uniqueness is guaranteed by the preceding paragraph).

Finally, set

$$
W=\left\{w \in \mathbf{R}^{N} \mid w^{i} \leq m^{i} \text { for some } i \in I\right\} .
$$

This is the set of all payoff vectors $w$ such that at least one player is better off by stopping at some stage rather than continuing forever and receiving $w$.

An important property of the set $W$ is that if the continuation payoff at stage $n$ is $w \in W$, and if player $i_{n}$ prefers to stop rather than continue (that is, $w^{i_{n}} \leq r_{n}^{i_{n}}$ ), then the expected payoff if player $i_{n}$ stops at stage $n$, $\left(1-p_{n}\right) w+p_{n} r_{n}$, is in $W$. Formally,

$$
w \in W \text { and } w^{i_{n}} \leq r_{n}^{i_{n}} \text { implies }\left(1-p_{n}\right) w+p_{n} r_{n} \in W \text {, for every } n \in \mathbf{N} \text {. }
$$

Indeed, under the assumptions, $\left(1-p_{n}\right) w^{i_{n}}+p_{n} r_{n}^{i_{n}} \leq r_{n}^{i_{n}} \leq m^{i_{n}}$, and (6) follows. 
Let $\varepsilon>0$ be given. We will prove the existence of a subgame perfect $\varepsilon$-equilibrium. We assume that $\varepsilon<\frac{1}{2} \min _{(i, r) \neq(j, \tilde{r})}\left|r^{i}-\tilde{r}^{i}\right|$. We also assume that $\varepsilon$ is small enough so that, for every $N \in \mathbf{N}$ and every finite sequence $\left(X_{1}, \ldots, X_{N}\right)$ of independent Bernouilli variables, with $\mathbf{P}\left(X_{n}=1\right)=p_{n}$, one has

$$
\mathbf{P}\left(\sup _{1 \leq n \leq N} X_{n}=1\right)<\varepsilon / 2 \Rightarrow \sum_{n=1}^{N} p_{n}<\varepsilon
$$

(see Rosenberg et al. (2002, Lemma 18) for a stronger statement).

We partition the set $\mathbf{N}$ of stages into infinitely many finite blocks. Set $n_{0}=1$ and, for $l \in \mathbf{N}$, define the initial stage $n_{l}$ of block $l$ to be

$$
n_{l}=\min \left\{n>n_{l-1} \mid \sum_{n_{l} \leq k<n,\left(i_{k}, r_{k}\right)=(i, r)} p_{k} \geq \epsilon \quad \forall(i, r) \in I R\right\} .
$$

Hence, in each block, all players have a probability at least $\epsilon$ to terminate the game with any vector they choose. Since $\pi(i, r)=+\infty$ for each $(i, r) \in I R$, all $n_{l}, l \in \mathbf{N}$, are finite.

Let $l>0$ be given. We will define a pure profile $\sigma=\sigma_{l}$ up to stage $n_{l}$. Later we will let $l$ vary. As for now, we omit the dependency of $\sigma_{l}$ on $l$. We will simultaneously construct a sequence $(w(n))_{n=1}^{n_{l}}$ of vectors in $W$. As a first approximation, the vector $w(n)$ may be interpreted as the expected payoff under $\sigma$ from stage $n$ onwards.

We define both $\sigma$ and $w$ backwards. We let $w\left(n_{l}\right)$ be an arbitrary point in $W \cap[-1,1]^{I}$. We deal with each of the blocks inductively (starting with the $l$ th one). Let $k \leq l$. Assuming $w\left(n_{k}\right) \in W$ is already defined, we define now $\sigma$ and $w$ over the stages $n=n_{k-1}, \ldots, n_{k}-1$.

Given $w(n+1)$ and $\sigma^{i_{n}}(n)$, we set $w(n)=\sigma^{i_{n}}(n) p_{n} r_{n}+\left(1-\sigma^{i_{n}}(n) p_{n}\right) w(n+$ $1)$, so that we need only define $\sigma^{i_{n}}(n)$. Thus, if $w(n+1)$ is the expected payoff from stage $n+1$ onward, $w(n)$ is the expected payoff from stage $n$ onward. Since $w\left(n_{l}\right) \in W$ and by $(6), w(n) \in W$ for every $n \leq n_{l}$.

Case $1 w^{i}\left(n_{k}\right) \leq m^{i}-\epsilon$ for some $i \in I$.

We define $\sigma$ by backward induction, with an appropriate tie-breaking rule. Set $\sigma^{i_{n}}(n)=1$ if $r_{n}^{i_{n}} \geq w^{i_{n}}(n+1)$, and $\sigma_{l}^{i_{n}}(n)=0$ otherwise.

Thus, at stage $n$, player $i_{n}$ compares his continuation payoff $w^{i_{n}}(n+1)$ to the payoff $r_{n}^{i_{n}}$ he would get by stopping, and he continues or stops accordingly. 
Case $2 w^{i}\left(n_{k}\right) \geq m^{i}-\epsilon$ for each $i \in I$.

Since $w\left(n_{k}\right) \in W$, there is a player $i_{*} \in I$ such that $w_{l}^{i_{*}}\left(n_{k}\right) \leq m^{i_{*}}$. At the final stages of the block only player $i_{*}$ will possibly stop. In earlier stages, $\sigma$ will be defined using backward induction as in Case 1.

Formally, let $n_{k-1} \leq n<n_{k}$. Assume that $\sigma$ has been defined for stages $q=n+1, \ldots, n_{k}-1$. We define $\sigma$ at stage $n$ as follows. Denote by $\pi\left(n+1, n_{k}\right)$ the probability under $\sigma$ that, starting from stage $n+1$, the game terminates under $\sigma$ before stage $n_{k}$, i.e.,

$\pi\left(n_{k}, n_{k}\right)=0$, and $\pi\left(q, n_{k}\right)=\sigma^{i_{q}}(q) p_{q}+\left(1-\sigma^{i_{q}}(q)\right) \pi\left(q+1, n_{k}\right)$ for $n+1 \leq q<n_{k}$.

Then:

- if $\pi\left(n+1, n_{k}\right)<\varepsilon$, we set $\sigma^{i_{n}}(n)=1$ if both $i_{n}=i_{*}$ and $r_{n}^{i_{*}} \geq w^{i_{*}}(n)$ hold. We set $\sigma^{i_{n}}(n)=0$ otherwise;

- if $\pi\left(n+1, n_{k}\right) \geq \varepsilon$, we set $\sigma^{i_{n}}(n)=1$ if $r_{n}^{i_{n}} \geq w_{l}^{i_{n}}(n)$, and $\sigma^{i_{n}}(n)=0$ otherwise.

We now prove that under $\sigma$, the probability of termination in any single block is bounded away from zero.

Lemma 7 For each $k$ such that $0 \leq k<l$, one has

$$
\mathbf{P}_{\sigma}\left(\theta<n_{k+1} \mid \theta \geq n_{k}\right) \geq \varepsilon / 3 \text {. }
$$

Proof. We consider Cases 1 and $\mathbf{2}$ in turn. In both cases, we will prove that $\pi\left(n_{k}, n_{k+1}\right) \geq \varepsilon / 3$.

We first assume that Case 1 holds, and we let $i_{*} \in I$ be a player such that $w^{i}\left(n_{k}\right) \leq m^{i}-\epsilon$.

1. If $\sigma^{i_{*}}(n)=1$ whenever $\left(i_{n}, r_{n}\right)=\left(i_{*}, \rho_{i_{*}}\right)$, one has $\pi\left(n_{k}, n_{k+1}\right) \geq \varepsilon / 2$, using (7) and since $\sum_{n: n_{k} \leq n<n_{k+1},\left(i_{n}, r_{n}\right)=\left(i_{*}, \rho_{i_{*}}\right)} p_{n} \geq \varepsilon$.

2. If $\sigma^{i_{*}}(n)=0$ for some $n$ such that $\left(i_{n}, r_{n}\right)=\left(i_{*}, \rho_{i_{*}}\right)$, then $w^{i_{*}}(n+1)>$ $m^{i_{*}}$. Observe now that, since payoffs are bounded by one, one has

$$
w^{i_{*}}(n+1) \leq \pi\left(n+1, n_{k+1}\right)+\left(1-\pi\left(n+1, n_{k+1}\right)\right) w^{i_{*}}\left(n_{k+1}\right) .
$$

This yields

$$
\pi\left(n+1, n_{k+1}\right) \geq \frac{\varepsilon}{1-m^{i_{*}}+\varepsilon} \geq \varepsilon / 3 .
$$

Since $\pi\left(n_{k}, n_{k+1}\right) \geq \pi\left(n+1, n_{k+1}\right)$, the conclusion also follows in that case. 
We next assume that Case $\mathbf{2}$ holds and we let $i_{*} \in I$ be the player distinguished in the definition of $\sigma$.

1. If $\sigma^{j}(n)=1$ for some $n$ and some player $j \neq i_{*}$, then as in item 2 above $\pi\left(n+1, n_{k+1}\right) \geq \varepsilon / 2$, hence $\pi\left(n_{k}, n_{k+1}\right) \geq \varepsilon / 2$.

2. If $\sigma^{j}(n)=0$ for each player $j \neq i_{*}$ and each stage $n$ with $i_{n}=j$, then $w^{i_{*}}(n) \leq m^{i_{*}}$ for each $n$. Indeed, only player $i_{*}$ stops, and his payoff is the average of $w^{i_{*}}\left(n_{k+1}\right) \leq m^{i_{*}}$ and $\rho_{i_{*}}^{i_{*}} \leq m^{i_{*}}$. Therefore $\sigma^{i_{*}}(n)=1$ whenever $\left(i_{n}, r_{n}\right)=\left(i_{*}, \rho_{i_{*}}\right)$, and one gets $\pi\left(n_{k}, n_{k+1}\right) \geq \varepsilon / 2$, as in item 1 above.

We will now let $l$ vary and we denote by $\sigma_{l}$ and $w_{l}$ the objects that were defined above. The pure strategy profile $\sigma_{l}$ may be identified with a point in $\{0,1\}^{\mathbf{N}}$ (the $n$th component being the behavior at stage $n$ of the active player $i_{n}$ ). Since the product space $\{0,1\}^{\mathbf{N}}$ is compact (and metrizable), the sequence $\left(\sigma_{l}\right)_{l \geq 0}$ has a subsequence that converges to some pure strategy profile $\sigma_{*}$. For notational convenience, we still denote this subsequence by $\left(\sigma_{l}\right)_{l \geq 0}$. Note that, for each $n \in \mathbf{N}$, the first $n$ components of $\sigma_{*}$ coincide with the first $n$ components of $\sigma_{l}$, provided $l$ is sufficiently large. For such $l$ 's, the behavior in the first $n$ stages of the game under the two strategy profiles $\sigma_{*}$ and $\sigma_{l}$ coincide.

Our goal is to prove that $\sigma_{*}$ is a subgame-perfect $\varepsilon$-equilibrium. We first prove that the play terminates $\mathbf{P}_{\sigma_{*}}$-a.s. in each subgame. We will then relate the payoff $\gamma\left(\sigma_{*}\right)$ to the sequence $\left(w_{l}\right)_{l \in \mathbf{N}}$ (Lemma 9$)$ and prove that no player has a profitable one-stage deviation (Lemma 10) under $\sigma_{*}$. The conclusion follows (Proposition 12), after we prove that no single player is responsible for the termination of the game (Lemma 11).

Corollary 8 For each $k \in \mathbf{N}$, one has

$$
\mathbf{P}_{\sigma_{*}}\left(\theta<n_{k+1} \mid \theta \geq n_{k}\right) \geq \varepsilon / 3 .
$$

Proof. Let $l \in \mathbf{N}$ be large enough so that $l>k$ and $\sigma_{*}$ coincides with $\sigma_{l}$ up to stage $n_{k+1}$, and apply Lemma 7 .

Lemma 9 For each $n \in \mathbf{N}$, one has

$$
\gamma_{n}\left(\sigma_{*}\right)=\lim _{l \rightarrow \infty} w_{l}(n) .
$$


Proof. We prove the result for $n=1$. The proof is similar for the subgame that starts at stage $n \in \mathbf{N}$.

Let $k \in \mathbf{N}$ be given. For each $l \in \mathbf{N}$, one has

$$
\begin{aligned}
& \gamma\left(\sigma_{*}\right)=\mathbf{E}_{\sigma_{*}}\left[r_{\theta} 1_{\theta<n_{k}}\right]+\mathbf{P}_{\sigma_{*}}\left(\theta \geq n_{k}\right) \gamma_{n_{k}}\left(\sigma_{*}\right), \text { and } \\
& w_{l}(1)=\mathbf{E}_{\sigma_{l}}\left[r_{\theta} 1_{\theta<n_{k}}\right]+\mathbf{P}_{\sigma_{l}}\left(\theta \geq n_{k}\right) w_{l}\left(n_{k}\right) .
\end{aligned}
$$

For $l$ large enough, the two profiles $\sigma_{l}$ and $\sigma_{*}$ coincide up to stage $n_{k}$. Hence, using Lemma 7 and Corollary 8, one gets, for $l$ large enough,

$$
\left\|\gamma\left(\sigma_{*}\right)-w_{l}(1)\right\| \leq 2\left(1-\frac{\varepsilon}{3}\right)^{k} .
$$

The result follows.

The next lemma says in substance that no player can increase his payoff by more than $3 \varepsilon$ by modifying his strategy in a single stage.

Lemma 10 Let $n \in \mathbf{N}$ be given. The following implications hold.

- If $\sigma_{*}^{i_{n}}(n)=0$ then $\gamma_{n+1}^{i_{n}}\left(\sigma_{*}\right) \geq r_{n}^{i_{n}}-3 \varepsilon$.

- If $\sigma_{*}^{i_{n}}(n)=1$ then $\gamma_{n+1}^{i_{n}}\left(\sigma_{*}\right) \leq r_{n}^{i_{n}}$.

Proof. Let $n \in \mathbf{N}$ be given. Let $l \in \mathbf{N}$ be sufficiently large so that $n_{l}>n$. We first prove a related statement for the strategy profile $\sigma_{l}$. Let $k<l$ be determined by $n_{k} \leq n<n_{k+1}$.

By construction, $\sigma_{l}^{i_{n}}(n)$ is defined using backward induction, except in some case where $\sigma_{l}^{i_{n}}(n)$ is required to be zero. In the former case, one has $\sigma_{l}^{i_{n}}(n)=1$ if $r_{n}^{i_{n}} \geq w_{l}^{i_{n}}(n+1)$ and $\sigma_{l}^{i_{n}}(n)=0$ otherwise. In the latter case, one has $w_{l}^{i_{n}}\left(n_{k+1}\right) \geq m^{i_{n}}-\varepsilon$ and $\pi\left(n+1, n_{k+1}\right)<\varepsilon$. Therefore, $\left|w_{l}^{i_{n}}\left(n_{k+1}\right)-w_{l}^{i_{n}}(n+1)\right|<2 \varepsilon$, which yields $w_{l}^{i_{n}}(n+1) \geq m^{i_{n}}-3 \varepsilon \geq r_{n}^{i_{n}}-3 \varepsilon$.

Hence, in both cases, one has

$$
\begin{array}{ll}
w_{l}^{i_{n}}(n+1) \leq r_{n}^{i_{n}} & \text { if } \sigma_{l}^{i_{n}}(n)=1, \text { and } \\
w_{l}^{i_{n}}(n+1) \geq r_{n}^{i_{n}}-3 \varepsilon & \text { if } \sigma_{l}^{i_{n}}(n)=0 .
\end{array}
$$

The conclusion follows by taking the limit $l \rightarrow+\infty$.

We now prove that the play terminates a.s., even if a single player chooses to continue whenever active. 
Lemma 11 For every $i \in I$ and every $n \in \mathbf{N}$, one has

$$
\mathbf{P}_{c^{i}, \sigma_{*}^{-i}}(\theta<+\infty \mid \theta \geq n)=1
$$

Proof. We argue by contradiction, and we assume that, for some player $i \in I$, the sequence $\mathbf{P}_{c^{i}, \sigma_{*}^{-i}}(\theta<+\infty \mid \theta \geq n)$ converges to zero when $n$ goes to $+\infty$. By Corollary 8 the game eventually terminates, so that $\mathbf{P}_{\sigma_{*}}(\theta<$ $+\infty \mid \theta \geq n)=1$ for every $n$. Therefore, it must be the case that player $i$ terminates the game: $\mathbf{P}_{\sigma_{*}^{i}, c^{-i}}(\theta<+\infty \mid \theta \geq n)=1$ for every $n$, and $\lim _{n \rightarrow+\infty}\left\|\gamma_{n}\left(\sigma_{*}\right)-\gamma_{n}\left(\sigma_{*}^{i}, c^{-i}\right)\right\|=0$.

We first prove that $\lim _{n \rightarrow+\infty} \gamma_{n}\left(\sigma_{*}\right)=\rho_{i}$, and then deduce a contradiction with the basic assumption made on $\Gamma$.

Step 1 : the sequence $\left(\gamma_{n}^{i}\left(\sigma_{*}\right)\right)_{n \in \mathbf{N}}$ is convergent.

Let $n \in \mathbf{N}$ be arbitrary. If $n \in N_{i}$ then Lemma 10 implies that $\gamma_{n}^{i}\left(\sigma_{*}\right) \geq$ $\gamma_{n+1}^{i}\left(\sigma_{*}\right)$. On the other hand, for $n \notin N_{i}$, one has $\gamma_{n}^{i}\left(\sigma_{*}\right)=\gamma_{n+1}^{i}\left(\sigma_{*}\right)$ if $\sigma_{*}^{i_{n}}(n)=0$, and

$$
\left|\gamma_{n}^{i}\left(\sigma_{*}\right)-\gamma_{n+1}^{i}\left(\sigma_{*}\right)\right|=p_{n}\left|r_{n}^{i}-\gamma_{n+1}^{i}\left(\sigma_{*}\right)\right| \leq 2 p_{n}
$$

if $\sigma_{*}^{i_{n}}(n)=0$.

Therefore, for every two positive integers $n \geq m$, one has

$$
\gamma_{m}^{i}\left(\sigma_{*}\right) \geq \gamma_{n}^{i}\left(\sigma_{*}\right)-2 \sum_{m \leq q<n ; q \notin N_{i}} p_{q} \mathbf{1}_{\sigma_{*}^{i q}(q)=1} .
$$

Let $\tilde{\varepsilon}>0$ be given. Assume that $\tilde{\varepsilon}$ is small enough so that (7) holds w.r.t. $\tilde{\varepsilon}$, and choose $N_{\tilde{\varepsilon}} \in \mathbf{N}$ sufficiently large so that $\mathbf{P}_{c^{i}, \sigma_{*}^{-i}}\left(\theta<+\infty \mid \theta \geq N_{\tilde{\varepsilon}}\right)<$ $\tilde{\varepsilon} / 2$. For such $N_{\tilde{\varepsilon}}, \sum_{N_{\tilde{\varepsilon} \leq q<+\infty ; q \notin N_{i}}} p_{q} \mathbf{1}_{\sigma_{*}^{i_{q}}(q)=1} \leq \tilde{\varepsilon}$. Therefore, by (8),

$$
\gamma_{m}^{i}\left(\sigma_{*}\right) \geq \gamma_{n}^{i}\left(\sigma_{*}\right)-2 \tilde{\varepsilon}, \text { for every } n \geq m \geq N_{\tilde{\varepsilon}} .
$$

This implies the convergence of $\left(\gamma_{n}^{i}\left(\sigma_{*}\right)\right)_{n \in \mathbf{N}}$, since it is a bounded sequence.

Step $2: \lim _{n \rightarrow+\infty} \gamma_{n}\left(\sigma_{*}\right)=\rho_{i}$.

Let $\lambda:=\lim _{n \rightarrow+\infty} \gamma_{n}^{i}\left(\sigma_{*}\right)$ be the limit of the payoffs to player $i$ in the successive subgames. We prove first that $\lambda=m^{i}$. Let $N \in \mathbf{N}$ be such that $\left|\gamma_{n}\left(\sigma_{*}\right)-\lambda\right|<\varepsilon / 2$ for each $n \geq N$. In particular, C1 or $\mathbf{C 2}$ below holds:

C1. $\gamma_{n}\left(\sigma_{*}\right)>m^{i}-\varepsilon$ for every $n \geq N$; 
C2. $\gamma_{n}\left(\sigma_{*}\right) \leq m^{i}-\varepsilon / 2$ for every $n \geq N$.

Assume first that $\mathbf{C 1}$ holds. By the choice of $\varepsilon$, one has $r_{n}^{i}<\gamma_{n+1}^{i}\left(\sigma_{*}\right)$ whenever $n \geq N, i_{n}=i$ and $r_{n} \neq \rho_{i}$. By Lemma 10, $\sigma_{*}^{i}(n)=0$ for each such stage. In other words, from stage $N$, player $i$ chooses to stop only in those stages such that $r_{n}=\rho_{i}$. Since $\mathbf{P}_{\sigma_{*}^{i}, c^{-i}}(\theta<+\infty \mid \theta \geq n)=1$ for each $n$, one therefore has $\gamma_{n}^{i}\left(\sigma_{*}^{i}, c^{-i}\right)=m^{i}$, for each $n \geq N$, which implies $\lambda=m^{i}$.

Assume next that $\mathbf{C 2}$ holds. We argue by contradiction and assume that $\lambda \neq m^{i}$. Since $\lambda=\lim _{n \rightarrow+\infty} \gamma_{n}^{i}\left(\sigma_{*}^{i}, c^{-i}\right)$, one has $\lambda<m^{i}$.

By Lemma 10, one has $\sigma_{*}^{i}(n)=1$ for each $n \geq N$ such that $\left(i_{n}, r_{n}\right)=$ $\left(i, \rho_{i}\right)$. We now adapt the argument of the first step and note that

$$
\gamma_{n}^{i}\left(\sigma_{*}\right)-\gamma_{n+1}^{i}\left(\sigma_{*}\right)=p_{n}\left(m^{i}-\gamma_{n+1}^{i}\left(\sigma_{*}\right)\right)
$$

for each such stage $n \geq N$. Therefore, using (8),

$\gamma_{N}^{i}\left(\sigma_{*}\right) \geq \gamma_{n}^{i}\left(\sigma_{*}\right)-2 \sum_{N \leq q<n: q \notin N_{i}} p_{q} \mathbf{1}_{\sigma_{*}^{i_{q}}(q)=1}+\sum_{N \leq q<n:\left(i_{q}, r_{q}\right)=\left(i, \rho_{i}\right)} p_{q}\left(m^{i}-\gamma_{q+1}^{i}\left(\sigma_{*}\right)\right)$,

a contradiction, since the second summation converges to $+\infty$ when $n$ goes to $+\infty$ while all other terms are bounded.

Hence, $\lim _{n \rightarrow+\infty} \gamma_{n}^{i}\left(\sigma_{*}\right)=m^{i}$. This yields $\lim _{n \rightarrow+\infty} \gamma_{n}^{i}\left(\sigma_{*}^{i}, c^{-i}\right)=m^{i}$, hence $\lim _{n \rightarrow+\infty} \gamma_{n}\left(\sigma_{*}^{i}, c^{-i}\right)=\rho_{i}$ since $\rho_{i} \in \mathbf{R}^{I}$ is the unique vector such that $(i, r) \in I R$ and $r^{i}=m^{i}$. Finally, this implies $\lim _{n \rightarrow+\infty} \gamma_{n}(\sigma)=\rho_{i}$.

Step 3 : The contradiction

By assumption, there exists $(j, \tilde{r}) \in I R$ such that $\tilde{r}^{j}>\rho_{i}^{j}$. Since $\mathbf{P}_{c^{i}, \sigma_{*}^{-i}}(\theta<$ $+\infty \mid \theta \geq m)<1$ for some $m \in \mathbf{N}$, and since $\pi(j, \tilde{r})=+\infty$, there are infinitely many stages $n$ such that $\left(i_{n}, r_{n}\right)=(j, \tilde{r})$ and $\sigma_{*}^{j}(n)=0$. For each such $n$, by Lemma 10, one has $\tilde{r}^{j}=r_{n}^{j}<\gamma_{n+1}^{j}\left(\sigma_{*}\right)$. Therefore, $\lim \sup _{n \rightarrow+\infty} \gamma_{n}^{j}\left(\sigma_{*}\right) \geq \tilde{r}^{j}$. Since $\tilde{r}^{j}>\rho_{i}^{j}=\lim _{n \rightarrow+\infty} \gamma_{n}^{j}\left(\sigma_{*}\right)$, we get a contradiction.

Proposition $12 \sigma_{*}$ is a subgame-perfect $3 \varepsilon$-equilibrium.

Proof. Let $i \in I$ be given. We prove that player $i$ cannot gain more than $3 \varepsilon$ by deviating from $\sigma_{*}$. The same proof will hold in any subgame, thereby showing the subgame-perfectness property.

Define the sequence $\left(X_{n}\right)_{n i n \mathbf{N}}$ of random variables by $X_{n}=r_{\theta}^{i}$ if $\theta<n$ and $X_{n}=\gamma_{n}^{i}\left(\sigma_{*}\right)$ if $\theta \geq n$. Let $\tau^{i}$ be an arbitrary strategy of player $i$. By 
Lemma 11, the sequence $\left(X_{n}\right)_{n i n \mathbf{N}}$ converges $\mathbf{P}_{\tau^{i}, \sigma_{*}^{-i}}$-a.s. to $X_{\infty}:=r_{\theta} \mathbf{1}_{\theta<+\infty}$ hence

$$
\lim _{n \rightarrow+\infty} \mathbf{E}_{\tau^{i}, \sigma_{*}^{-i}}\left[X_{n}\right]=\mathbf{E}_{\tau^{i}, \sigma_{*}^{-i}}\left[r_{\theta} \mathbf{1}_{\theta<+\infty}\right]=\gamma^{i}\left(\tau^{i}, \sigma_{*}^{-i}\right) .
$$

On the other hand, by Lemma 10 , one has $\mathbf{E}_{\tau^{i}, \sigma_{*}^{-i}}\left[X_{n+1} \mid \mathcal{H}_{n}\right] \leq X_{n}+$ $3 \varepsilon \mathbf{1}_{\theta=n}$, where $\mathcal{H}_{n}$ is the past play up to stage $n$. By taking expectations, and by summation over $n$, one obtains $\lim _{n \rightarrow+\infty} \mathbf{E}_{\tau^{i}, \sigma_{*}^{-i}}\left[X_{n}\right] \leq X_{0}+3 \varepsilon$ which yields, using (9),

$$
\gamma^{i}\left(\tau^{i}, \sigma_{*}^{-i}\right) \leq \gamma^{i}\left(\sigma_{*}\right)+3 \varepsilon
$$

\section{Discussion}

A natural extension of the model is to drop the assumption that the order of the players is deterministic. That is, to assume that $\left(i_{n}, p_{n}, r_{n}\right)_{n \in \mathbf{N}}$ is a (non-deterministic) process. Whether or not there is a subgame-perfect $\varepsilon$-equilibrium in this model is still unknown.

When $|I|=2$, one can generalize the result of Shmaya and Solan (2002) to show that an $\varepsilon$-equilibrium exists. However, it is not known whether pure subgame-perfect $\varepsilon$-equilibrium profiles in Markovian strategies exist.

One can even restrict oneself to the following simple class of games. Let $I$ be a finite set of players, and, for each $i \in I$, fix $r_{i} \in \mathbf{R}^{I}$. At every stage, independent of past play, a player $i \in I$ is chosen at random, each player is chosen with probability $1 /|I|$. Player $i$ then has to decide whether he stops or continues. If he continues, the game continues to the next stage, while if he stops, the game terminates with probability 1 , and the terminal payoff is given by $r_{i}$.

Whether or not any such game admits a subgame-perfect $\varepsilon$-equilibrium in Markovian strategies is an open problem, which seems to have significant implications on the study of equilibrium payoff in stochastic games. 


\section{References}

[1] Dynkin E.B. (1969) Game Variant of a Problem on Optimal Stopping, Soviet Math. Dokl., 10, 270-274

[2] Fine C.H. and Li L. (1989) Equilibrium Exit in Stochastically Declining Industries. Games Econ. Behavior, 1, 40-59.

[3] Flesch J., Thuijsman F. and Vrieze K. (1997) Cyclic Markov Equilibria in Stochastic Games, Int. J. Game Th., 26, 303-314

[4] Fudenberg D. and Tirole J. (1991) Game Theory, The MIT Press

[5] Ghemawat P. and Nalebuff B. (1985) Exit, RAND J. Econ., 16, 184-194

[6] Kiefer Y.I. (1971) Optimal Stopped Games, T. Prob. Appl., 16, 185-189

[7] Kilgour D.M. (1975) The Sequential Truel, Int. J. Game Theory, 4, 151174

[8] Kilgour D.M. (1977) Equilibrium Points in Infinite Sequential Truels, Int. J. Game Theory, 6, 167-180

[9] Kilgour D.M. and Brams S.J. (1997) The Truel, Math. Magazine, 70, 315-325

[10] Martin D.A. (1975) Borel Determinacy, Ann. Math. (2) 102, 363-371

[11] Mertens, J.F. (1987) Repeated Games, Proceedings of the International Congress of Mathematicians, Berkeley, California, 1528-1577

[12] Neveu J. (1975) Discrete-Parameter Martingales, North-Holland, Amsterdam

[13] Rosenberg D., Solan E. and Vieille N. (2001) Stopping Games with Randomized Strategies, Prob. Th. Related Fields, 119, 433-451

[14] Rosenberg D., Solan E. and Vieille N. (2002) On the MaxMin Value of Stochastic Games with Imperfect Monitoring, Discussion Paper 1337, The Center for Mathematical Studies in Economics and Management Science, Kellogg School of Management, Northwestern University 
[15] Shmaya E. and Solan E. (2002) Two-Player Non-Zero-Sum Stopping Games in Discrete Time, Discussion Paper 1347, The Center for Mathematical Studies in Economics and Management Science, Kellogg School of Management, Northwestern University

[16] Solan E. and Vieille N. (2001), Quitting Games, Math. Oper. Res., 26, 265-285 